\title{
Better vascular function tests in cardiovascular care: learning from evidence and providing improved diagnostics to the patient
}

\author{
Atsushi Tanaka ${ }^{1} \cdot$ Koichi Node ${ }^{1}$ \\ Received: 28 November 2021 / Accepted: 30 November 2021 / Published online: 27 December 2021 \\ (C) The Japanese Society of Hypertension 2021
}

In hypertensive organ disorders, it goes without saying that adverse effects occur mainly in blood vessels. High blood pressure causes damage to vascular structure and function, eventually leading to vascular dysfunction and failure. When treating hypertension, it is therefore necessary not only to evaluate blood pressure levels but also to comprehensively evaluate organ damage, including degradation of vascular function. In the Japanese Society of Hypertension Guidelines for the Management of Hypertension (JSH 2019) [1], the evaluation of hypertensive vascular disorders, including atherosclerosis, can be divided broadly into two types: morphological and functional assessment. Morphological assessment is performed by various imaging modalities, including ultrasound, while functional assessment is performed by physiological tests, such as the vascular endothelial function test, pulse wave velocity (PWV), and pulse wave analysis. Although differences in the measurement principles and the clinical significance of the results of each test are beyond the scope of our review, all the physiological tests have been studied extensively, and relevant meta-analyses have shown that they all have good prognostic value.

On the other hand, it is questionable whether these tests have been accurately interpreted and fully utilized in an integrated way to improve the quality of care for hypertension patients. If they have not, there may have been some clinical barriers, such as availability and cost-effectiveness. In addition to technical issues, such as convenience and reproducibility, there is also limited evidence and consensus on clear cutoff values and on the relationship between improvement in indices and treatment and clinical prognosis. These issues may have made it difficult to apply the

Atsushi Tanaka

tanakaa2@cc.saga-u.ac.jp

1 Department of Cardiovascular Medicine, Saga University, Saga, Japan results to daily clinical assessment of patients, thereby negatively affecting the clinical use of the tests. We believe it is important that tests can be applied to actual clinical situations to improve the quality of patient management.

The '5-Year Plan for Overcoming Stroke and Cardiovascular Disease' proposed by the Japan Stroke Society and the Japanese Circulation Society in 2016 stated that more action is urgently required to advance preemptive medicine using biomarkers and vascular function tests [2]. To promote such action, it is clear that the aforementioned challenges of vascular function testing must be overcome. Therefore, we carried out a review of past literature and systematically organized physiological vascular function tests to propose normal and abnormal ranges for several tests based on the assumption that vascular failure is the foundation of cardiovascular disease [3].

Vascular failure, proposed by Drs. Inoue and Node in 2006 as an entity integrating multiple forms of vascular disease, includes a broad spectrum of vascular damage, injury, and dysfunction [4]. This concept consists of three major pathophysiological conditions: vascular endothelial dysfunction, vascular smooth muscle dysfunction, and vascular metabolic dysfunction. The physiological diagnostic criteria for vascular failure are based on target vascular layers and areas. Two tests (flow-mediated vasodilation [FMD] and reactive hyperemia peripheral arterial tonometry) are used to assess endothelial function, while two tests (PWV and the cardio-ankle vascular index [CAVI]) are used to evaluate arterial stiffness, a marker indicative of medial layer function [3]. Two cutoff values for each test were proposed with the following aims: (i) to separate the normal and borderline ranges associated with conventional cardiovascular risk factors and (ii) to separate the borderline and abnormal ranges associated with an increased risk of cardiovascular disease [3, 5]. For the details of these criteria, please refer to the relevant publications [3, 5].

The physiological vascular function tests included in the criteria for vascular failure were selected from diagnostic 
tools that are widely available in daily clinical practice. On the other hand, other vascular tests were excluded from these diagnostic criteria due mainly to several academic and clinical reasons, such as the volume of evidence and its dissemination in actual clinical practice. However, we understand that debate remains as to which test should be considered the gold standard for diagnosing vascular function and vascular failure. Furthermore, there is no clear clinical consensus on whether a combination of multiple tests improves diagnostic performance compared to a single test. It is possible that simultaneous application of different vascular tests may further stratify the risk of cardiovascular events [6]. However, there is always a question as to which is the optimal test in daily clinical practice for evaluating and monitoring vascular function and damage.

Another question is which criteria should be used for selecting these tests. One practical approach may be to use the test available at the hospital. We fully understand that test availability can limit the range of clinical options, although tests selected in this manner may not be optimal in terms of their underlying clinical significance. If only one option is available, then the discussion must end for the time being. Otherwise, in addition to considering the principles and vascular properties underlying each test, it is important to make a direct comparison of the diagnostic and prognostic capabilities of different tests, as it would be expected to provide insights to guide the selection of a test in daily clinical practice.

In this issue of the journal, Ishida et al. [7] performed a cross-sectional study in patients with treated essential hypertension to compare the associations of CAVI and the augmentation index (AI) with relevant cardiovascular risk markers. The participants underwent both of these vascular tests within a period of less than three months and received other vascular tests to measure FMD and carotid intimamedia thickness (CIMT). The key findings of the study were as follows: (i) CAVI was more closely associated than AI with relevant cardiovascular risk markers; (ii) CAVI correlated positively with both FMD and CIMT values, whereas AI did not; and (iii) these findings were almost entirely consistent across age and sex. The findings suggested that CAVI was more suitable than AI for reflecting background cardiovascular risk status and systemic and local burdens of arteriosclerosis. This may partly support the preferential measurement of CAVI in daily clinical practice and the inclusion of this test in the diagnostic criteria for vascular failure. At the same time, three large-scale observational studies using CAVI in Japan showed a detailed relationship between this variable and cardiovascular risk factors and confirmed its predictive value for cardiovascular events [8-10]. On the other hand, the precise reason(s) why AI tests showed a weaker association with these parameters remains uncertain; possible explanations include differences in the principles of these two tests and the vascular properties they measure.

Given these intriguing results and the relative ease of use of the device involved, CAVI will become a strong candidate as a vascular function test for preemptive medicine. Notably, the logic of the speculation by Ishida and colleagues appears to be that CAVI is a better tool for determining atherosclerotic vascular properties mainly due to its stronger association with relevant risk factors and parameters. In fact, such a close linkage would be clinically useful for evaluating the current burden of cardiovascular risk and, to some extent, the future risk of cardiovascular events. However, is it possible to select test devices based on these associations alone? In other words, the purpose of performing vascular function tests is called into question. The ultimate purposes of vascular function testing in individual patients are to accurately identify any pathophysiological conditions affecting vascular function, to evaluate the risk of adverse cardiovascular events and prognosis, and to implement optimal therapy based on the level of risk measured in the patient. However, there are still several clinical concerns related to vascular function tests: (1) it is difficult to understand the tests in a systematic way due to confusion regarding several of the tests and methods; (2) the appropriateness of the tests in various clinical situations remains unclear; (3) the tests may be easily influenced by various factors, such as aging and comorbidities, making it difficult to set strict standard values; and (4) little evidence has been accumulated regarding efficient therapeutic intervention methods based on vascular function tests. A clinically reliable vascular function test is therefore likely to require multitasking. At the same time, clinicians need to correctly understand the vascular properties of each test and select the appropriate test according to the clinical situation of the patient. Therefore, the clinical utility of vascular function tests from a multifaceted point of view needs to be validated and then applied to individual patients in everyday clinical settings (Fig. 1).

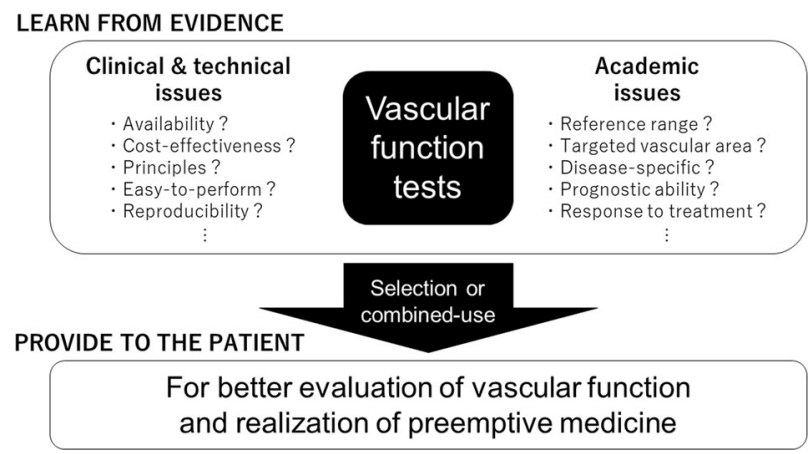

Fig. 1 To improve vascular function testing in cardiovascular care, clinicians should learn from evidence and provide up-to-date, evidence-based diagnostics to the patient 
In the journey of vascular research, we hope that researchers and clinicians will elucidate the theoretical and practical mysteries behind vascular function tests and realize improved patient-centered preemptive medicine that utilizes improved vascular function tests in cardiovascular care.

Acknowledgements The authors would like to express their gratitude to the members of the Physiological Diagnosis Criteria for Vascular Failure Committee.

\section{Compliance with ethical standards}

Conflict of interest The authors declare no competing interests.

Publisher's note Springer Nature remains neutral with regard to jurisdictional claims in published maps and institutional affiliations.

\section{References}

1. Umemura S, Arima H, Arima S, Asayama K, Dohi Y, Hirooka Y, et al. The Japanese Society of Hypertension Guidelines for the Management of Hypertension (JSH 2019). Hypertens Res. 2019;42:1235-481.

2. http://www.j-circ.or.jp/five_year/files/five_year_plan.pdf (in Japanese) accessed on November 26, 2021.
3. Tanaka A, Tomiyama H, Maruhashi T, Matsuzawa Y, Miyoshi T, Kabutoya T, et al. Physiological diagnostic criteria for vascular failure. Hypertension 2018;72:1060-71.

4. Inoue T, Node K. Vascular failure: a new clinical entity for vascular disease. J Hypertens. 2006;24:2121-30.

5. Tanaka A, Tomiyama H, Maruhashi T, Matsuzawa Y, Miyoshi T, Kabutoya $\mathrm{T}$, et al. Official announcement of physiological diagnostic criteria for vascular failure from the Japanese society for vascular failure. Vasc Fail. 2018;2:59-60.

6. Tajima E, Sakuma M, Tokoi S, Matsumoto H, Saito F, Watanabe $\mathrm{R}$, et al. The comparison of endothelial function between conduit artery and microvasculature in patients with coronary artery disease. Cardiol J. 2020;27:38-46.

7. Ishida $\mathrm{K}$, Morimoto $\mathrm{S}$, Horiuchi $\mathrm{S}$, Kimura $\mathrm{M}$, Ishikawa $\mathrm{T}$, Kimura S, et al. Comparison of the usefulness of cardio-ankle vascular index and augmentation index as an index of arteriosclerosis in patients with essential hypertension. Hypertens Res. 2021. https://doi.org/10.1038/s41440-021-00823-x.

8. Tabara Y, Setoh K, Kawaguchi T, Takahashi Y, Kosugi S, Nakayama $\mathrm{T}$, et al. Factors affecting longitudinal changes in cardio-ankle vascular index in a large general population: the Nagahama study. J Hypertens. 2018;36:1147-53.

9. Kabutoya T, Hoshide S, Fujiwara T, Negishi K, Nishizawa M, Yamamoto M, et al. Age-related difference of the association of cardiovascular risk factors with the cardio-ankle vascular index in the Cardiovascular Prognostic Coupling Study in Japan (the Coupling Registry). J Clin Hypertens. 2020;22:1208-15.

10. Miyoshi T, Ito H, Shirai K, Horinaka S, Higaki J, Yamamura S, et al. Predictive value of the cardio-ankle vascular index for cardiovascular events in patients at cardiovascular risk. J Am Heart Assoc. 2021;10:e20103. 